\title{
Sensitive Measures of Condition Change in EEG Data*
}

\author{
${ }^{\dagger}$ L. M. Hively, ${ }^{\mp}$ P. C. Gailey, and ${ }^{\ddagger}$ V. A. Protopopescu \\ ${ }^{\dagger}$ Engineering Technology Division \\ Energy Division \\ ${ }^{\ddagger}$ Computer Science and Mathematics Division \\ Oak Ridge National Laboratory \\ P. O. Box 2008, Bldg. 6025 \\ Oak Ridge, TN 37831-6364
}

\begin{abstract}
"This submitted manuscript has been authored by a contractor of the U. S. government under Contract No. DE-AC05-98OR22464. Accordingly, the U. S. Government retains a nonexclusive, royalty-free license to publish or reproduce the published form of this contribution, or allow others to do so, for U. S. Government purposes."
\end{abstract}

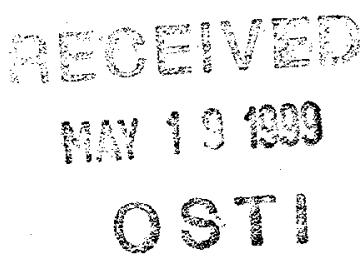

Submitted to: Proceedings of the International Conference "Chaos in Brain?," Bonn, Germany, March 10-12, 1999.

*Research supported by the U. S. Department of Energy, Office of Energy Management and Office of Basic Energy Sciences, under contract No. DE-AC05-96OR22464 with Lockheed Martin Energy Research Corporation. 


\section{DISCLAIMER}

This report was prepared as an account of work sponsored by an agency of the United States Government. Neither the United States Government nor any agency thereof, nor any of their employees, make any warranty, express or implied, or assumes any legal liability or responsibility for the accuracy, completeness, or usefulness of any information, apparatus, product, or process disclosed, or represents that its use would not infringe privately owned rights. Reference herein to any specific commercial product, process, or service by trade name, trademark, manufacturer, or otherwise does not necessarily constitute or imply its endorsement, recommendation, or favoring by the United States Government or any agency thereof. The views and opinions of authors expressed herein do not necessarily state or reflect those of the United States Government or any agency thereof. 


\section{DISCLAIMER}

Portions of this document may be illegible in electronic image products. Images are produced from the best available original document. 


\title{
SENSITIVE MEASURES OF CONDITION CHANGE IN EEG DATA
}

\author{
L. M. HIVELY, P. C. GAILEY, AND V.A. PROTOPOPESCU \\ Oak Ridge National Laboratory \\ P.O. Box 2009, Oak Ridge, TN 37831-8066 \\ E-mail: hivelylm@ornl.gov; gaileypc@ornl.gov; protopopesva@ornl.gov
}

\begin{abstract}
We present a new, robust, model-independent technique for measuring condition change in nonlinear data. We define indicators of condition change by comparing distribution functions (DF) defined on the attractor for time windowed data sets via. $L_{1}$-distance and $\chi^{2}$ statistics. The new measures are applied to EEG data with the objective of detecting the transition between non-seizure and epileptic brain activity in an accurate and timely manner. We find a clear superiority of the new metrics in comparison to traditional nonlinear measures as discriminators of condition change.
\end{abstract}

The aim of this paper is twofold: (i) we present a new, robust, modelindependent technique for measuring condition change in nonlinear data and (ii) we apply it to clinical EEG data. Our technique focuses on discriminating between different and possibly close chaotic regimes.

Based on the phase space (PS) reconstruction of the dynamics from delayed time series ${ }^{1}$ various nonlinear measures have been defined to characterize process dynamics. We choose three of these nonlinear measures, against which to compare the new metrics. In particular, we use ${ }^{1}$ : (i) the first minimum in the mutual information function, $M_{1}$, as a measure of decorrelation time, (ii) the correlation dimension, $D$, as a measure of dynamic complexity, and (iii) the Kolmogorov entropy, $K$, as a measure of predictability.

While describing certain global features of the nonlinear dynamics, these traditional nonlinear measures do not capture the host of finer details that could be responsible for condition change. The same is true for other global indicators such as fractal dimension, Lyapunov exponents, etc. The main reason for this insufficient discriminating power is the fact that in the definition of these indicators most dynamical details cancel each other out by averaging over many cycles. In order to capture these minute details, more refined indicators are needed. We define two such new indicators starting from the invariant measure of the dynamics on the attractor. We represent the distribution function (DF) on the attractor by using $N$ discrete cells. We denote the population in the $i$-th PS-DF state as $Q_{i}$ for the basecase and $R_{i}$ for the unknown and we measure the difference between $Q_{i}$ with $R_{i}$ by the $\chi^{2}$ statistics and $L_{1}$ distance:

$$
\begin{gathered}
\chi^{2}=\sum_{i=1}^{N}\left(Q_{i}-R_{i}\right)^{2} /\left(Q_{i}+R_{i}\right), \\
L=\sum_{i=1}^{N}\left|Q_{i}-R_{i}\right| .
\end{gathered}
$$


The choice of these measures is dictated by the following considerations. The $\chi^{2}$ statistics is one of the most powerful, robust, and widely-used statistical tests to measure discrepancies between observed and expected frequencies. The $L_{1}$ distance is the natural metric for DFs since it is directly related to the total invariant measure on the attractor. To apply these measures properly we have to scale the total population of the unknown DF (sum over all the domain populations in $R_{i}$ ) to be the same as the total population of the basecase.

By connecting successive PS points as indicated by the dynamics, $y(i) \rightarrow y(i+1)$, one obtains a discrete representation of the process flow ${ }^{1}$. This approach motivates the extension of the PS method to capture even more dynamical information using pair-wise connectivity between successive $d$-dimensional PS states, thus forming a $2 d$-dimensional vector, $Y(i)=[y(i), y(i+1)]$, in the connected-PS (CPS). The connected distribution functions (CDF) are $Q_{i j}$ and $R_{i j}$ for the basecase and unknown processes, respectively. The index, $i$, denotes the beginning ( $i$-th) PS state, and $j=i+1$ denotes the ending ( $j$-th) PS state. We define the connected $\chi^{2}$ statistic, $\chi_{c}^{2}$, and the connected $L_{1}$ distance, $L_{c}$, by expressions similar to Eqs. (1)-(2) in which we replace $Q_{i}, R_{i}$ by $Q_{i j}, R_{i j}$ and sum over both indices, $i, j=1, \ldots N$. These measures satisfy the following inequalities: (i) $\chi^{2} \leq L$; (ii) $\chi_{c}^{2} \leq L_{c}$; (iii) $L \leq L_{c}$; and (iv) $\chi^{2} \leq \chi_{c}^{2}$, which show that, as expected, the measures of condition change for the CPS are stronger than those for the PS representation.

We applied these measures to brain wave data, which have often been described in terms of nonlinear dynamics ${ }^{2}$. Nonlinear EEG measures are not stationary, displaying marked transitions between normal and epileptic states. EEG data display clear PS structure and low-dimensional features ${ }^{2,3}$ with at least one positive Lyapunov exponent ${ }^{2,4}$ and hence positive Kolmogorov entropy. Thus, use of the new $L_{1}$ and $\chi^{2}$ PS and CPS measures seems warranted for detection of nonlinear condition changes in brain wave data. Since brain dynamics in EEG are obscured by muscular activity due to eye blinks, facial twitches, etc., we eliminated by using a zero-phase quadratic filter. ${ }^{5,6}$.

Figure 1 shows a typical plot of nonlinear EEG measures versus time. For the first 800 seconds, variability in all of the measures is modest, representing the dynamics of normal brain activity. The clinical seizure occurs at 2620-2726 seconds, as indicated by the vertical bars at these times in this (and the subsequent) figure. A pre-seizure indication in $D$ occurs as a small decrease at 1150 seconds (Fig. 1a). Likewise, a pre-seizure indication in $K$ is a small peak at 1150 seconds (Fig. 1b). Later variations in $D$ and $K$ provide no clear pre-seizure indication in Fig. 1. The value of $M_{1}$ rises slowly from 12 to 20 during the non-seizure period $(0-1100$ seconds), then falls gradually to an average value of 14 (range: 11-20) during the pre-seizure period (Fig. 1c). During the seizure, $D$ and $K$ show clear peaks; while $M_{1}$ falls abruptly. In sharp contrast to these weak pre-seizure indications, clear peaks occur in the PS measures (Figs. 1d-g) at 1150, 1300, 1600, and 2350 seconds.

Our approach differs markedly from earlier work ${ }^{3,4}$ in several respects. First, previous investigations used data from subdural and depth electrodes, while we use scalp EEG data that allows non-invasive, ambulatory, long-term monitoring. Second, those studies obtained EEG data from the epileptogenic area, while we used data only from channel 13 in the bipolar montage, showing the robustness of 
our method, and allowing easy electrode placement by a patient for non-clinical monitoring. Third, prior effort used invasive monitoring to avoid low-frequency artifacts, which we removed from scalp data with a zero-phase quadratic filter, dramatically improving the data quality and the nonlinear measures. Fourth, previous investigations focused only on temporal lobe epilepsy, while we earlier determined no consistent trends in $D$ and $K$ for various seizure types and instead focused on these new measures of condition changes for any seizure. These advantages will facilitate routine use by patients in a non-clinical setting.

Practical use of this method for forewarning epileptic events will require characterization of several seizures for each patient, and detailed determination of detection criteria. Future work will involve: (i) statistical evaluation of false positives and negatives in epileptic patients, and of false positives in normal patients and (ii) clinical monitoring of each patient to determine optimal (C)PS construction parameters, which subsequently would be fixed for ambulatory monitoring.

We presented two new model-independent measures to detect non-stationarity (condition change) in nonlinear time series. These indicators integrate (and magnify) the differences between the process dynamics, and avoid the inner cancellation effects due to averaging over many orbits. The new measures also indicate significant pre-seizure changes in all nine analyzed EEG datasets $521-2200$ seconds before the clinical seizure while correlation dimension, Kolmogorov entropy, and mutual information fail to detect any change at all in three, two, and five EEG datasets, respectively ${ }^{6}$. These results show that the new measures are superior to traditional nonlinear measures for detection of condition change.

\section{Acknowledgments}

This work was sponsored by the U. S. Department of Energy, Office of Energy Management. V. P. was partially supported by the U. S. Department of Energy, Office of Basic Energy Sciences. Oak Ridge National Laboratory is managed for the United States Department of Energy by Lockheed Martin Energy Research Corporation, under Contract No. DE-AC05-96OR22464.

\section{References}

1. H.D.I. Abarbanel, Analysis of Observed Chaotic Data, Springer Publ., New York (1996).

2. T. Elbert, W.J. Ray, Z.J. Kowalik, J.E. Skinner, K.E. Graf, and N. Birbaumer, Physiol. Rev., 74, 1 (1994).

3. K. Lehnertz and C.E. Elger, Phys. Rev. Lett., 80, 5019 (1998).

4. L.D. Iasemidis and J.C. Sackellares, Neuroscientist, 2, 118 (1996).

5. L.M. Hively, N.E. Clapp, C.S. Daw, and W.F. Lawkins, ORNL/TM-12961 (Oak Ridge National Laboratory, Oak Ridge, TN) 1995.

6. L. M. Hively, P. C. Gailey, and V. A. Protopopescu, submitted to Phys. Lett. A. 
Figure 1. Nonlinear measures versus time for dataset \#62723t: (a) correlation dimension, $D$, (b) Kolmogorov entropy, $K$, (c) first minimum in the MIF $M_{1}$, (in timesteps), (d) $L_{c}$, (e) $L$, (f) $\chi_{c}^{2}$, and (g) $\chi^{2}$. 

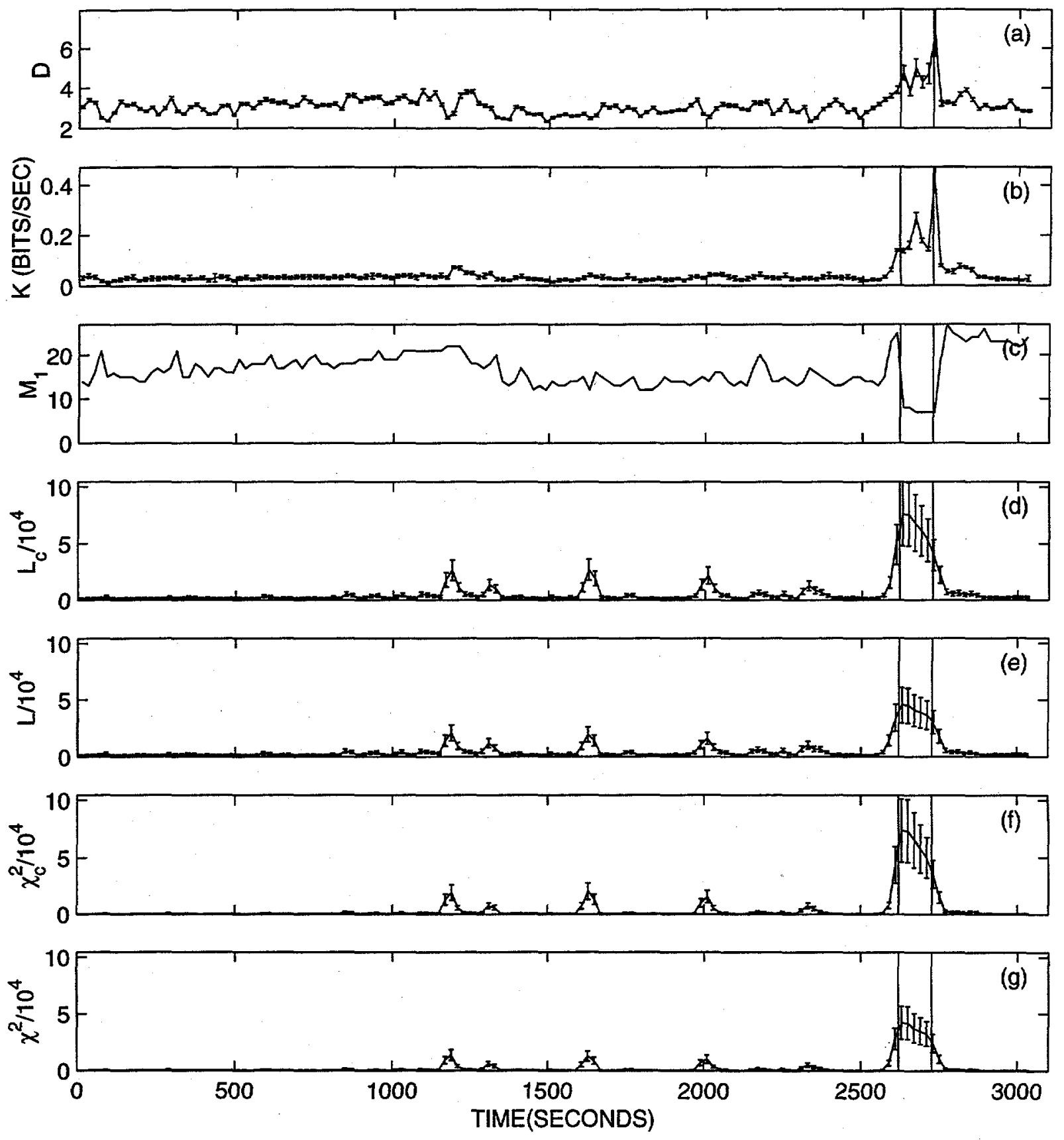